\title{
Development of a Palm Portable Mass Spectrometer
}

\author{
Mo Yang, Tae-Young Kim, Hyun-Chul Hwang, Seok-Kyung Yi, \\ and Do-Hoon Kim \\ Analytical Instrumentation Research Institute, Sam Yang Chemical Company, Seoul, Korea
}

\begin{abstract}
A palm portable mass spectrometer (PPMS) has been developed with a weight of $1.48 \mathrm{~kg}(3 \mathrm{lb})$ and a size of $1.54 \mathrm{~L}\left(8.2 \times 7.7 \times 24.5 \mathrm{~cm}^{3}\right)$ that can be operated with an average battery power of $5 \mathrm{~W}$. A miniaturized ion trap has been used as a mass analyzer that consists of four parallel disks with coaxial holes. A rf voltage of $1500 \mathrm{~V}_{p-p}$ at $3.9 \mathrm{MHz}$ has been used for scanning ion mass of up to $\mathrm{m} / z$ 300. An ion-getter pump serves for high vacuum of the PPMS. Sample gas was introduced in pulse mode. An embedded microcomputer has been developed for system control. Detection of organic gases diluted in the air has been demonstrated up to $6 \mathrm{ppm}$ for toluene and $22 \mathrm{ppm}$ for dimethyl methylphosphonate (DMMP). Performance results suggest usefulness of the PPMS as a personal mobile device for detection/identification of chemical warfare agents in the field. $\mathrm{J}$ Am Soc Mass Spectrom 2008, 19, 1442-1448) @ 2008 American Society for Mass Spectrometry
\end{abstract}

$\mathrm{T}$ The development of portable chemical analyzers has been of great interest for applications such as the detection and identification of chemical warfare agents (CWA) in the field, the monitoring of air pollution and environmental quality, the inspection of explosives and drugs by homeland security, and the analysis of gases during space exploration. Although the mass spectrometer is one of the most powerful tools of chemical analysis, its use in the field as a portable device has been limited mainly because of its size, weight, and power requirements. For this reason, alternative methods have been widely used for chemical agent detection in the field [1-3], e.g., ion mobility spectrometry (IMS), flame photometry, infrared spectroscopy, electrochemistry, color change chemistry, surface acoustic wave, photo-ionization, conductive polymer, flame-ionization, etc. The basic concept of IMS is to characterize chemical substances through gas-phase ion mobility at atmospheric pressure [4]. An advantage of IMS is that it does not need a vacuum pump, and so its weight and size can be reduced. However, a disadvantage is the poor resolution and broadening of the ion flight time distribution caused by ions continually colliding with air molecules. For this reason, the rate of false alarms is high when other chemical substances are present in the background. Another disadvantage is the need for an intense radiation source for ionization at atmospheric pressure, which can give rise to environmental issues. Other devices using surface acoustic waves or conductive polymers are very compact in size, but these do not display spectrum-based information of

Address reprint requests to Dr. M. Yang, Analytical Instrumentation Research Institute, Sam Yang Chemical Co., Seoul, Korea. E-mail: dryangmo@yahoo.com unknown gases [5], and can only be used for confirming the presence of a specific group of agents. In the field, the capability for not only detection but also identification is very important because specific identification of a poison gas is needed for immediate detoxification of victims. However, the identification of chemical substances in the field with a personal mobile device has not yet been successfully achieved.

A mass spectrometer is uniquely able to differentiate chemical substances based on molecular mass spectra. A number of mass spectrometers have been developed for military use, such as MM1 [1] and CBMS [6]. However, these are large and heavy, and are deployed only as vehicle mounted instruments. Cooks et al. have reported a Mini-10 handheld ion trap mass spectrometer [7] that weighs only $10 \mathrm{~kg}$. A mass spectrometer based helium leak detector has been developed by MKS Instrument Inc. that reduces device weight to $7 \mathrm{~kg}$ [8]. However, a portable mass spectrometer as a personal mobile chemical analyzer for field use requires further reductions in size and weight.

The target gases of a chemical analyzer for military use are mainly nerve agents, choking agents, blood agents, and blister agents [9]. The mass range of the portable mass spectrometer should be up to $300 \mathrm{~m} / \mathrm{z}$, since the molecular weights of the most significant agents are in this range. The mass resolution should be high enough to resolve the agents from background interference. The detection limit should be lower than one tenth of the concentration level at which self-contained breathing apparatus or respirators must be worn or immediate life threatening effects will occur [2]. Response time, also called detection time, should be as fast as possible because a faster alarm reduces the inhalation of contaminated air before donning 
of protective equipment and evacuation from the contaminated environment. Monitoring of air contamination from a fast moving vehicle or aircraft also requires a fast detection response time.

A large number of groups have attempted to miniaturize a mass spectrometer using ion trap, quadrupole, FTICR, time-of flight, and sector field mass analyzers $[10,11]$. An ion trap mass analyzer has several advantages over the other types. First, it can operate under relatively low vacuum, reducing the pumping load of the vacuum system. Second, it scans all ions of interest in a single pulse of ionization, quickly capturing information about chemical composition even when the sample concentration is rapidly changing. Another advantage is that miniaturization of the ion trap does not sacrifice performance in mass resolution or mass range. For example, Whitten et al. [12] have demonstrated a submillimeter size ion trap that resolves the isotope ratio of xenon, while Cooks et al. have developed a micron size cylindrical ion trap [13]. Small size also reduces trapping capacity because of ion repulsion forces, and a multi-array of micro ion traps in various dimensions has been proposed to compensate for this loss of sensitivity [14, 15].

The most difficult part of building a portable mass spectrometer is a high vacuum system in a small size appropriate to the miniaturized mass analyzer. Its pumping speed must be high enough to evacuate the system in a tolerable time and maintain the high vacuum while the air sample is introduced. Its power consumption should be minimal to allow extended operation with a small battery.

Miniaturization of various high vacuum pumps has been reported, including turbo-molecular pumps, cryogenic pumps, and ion-getter pumps. Ion getter pumps and cryogenic pumps have the advantage that they can operate without a forestage roughing pump. An ion getter pump is preferable because it has no mechanical motion.

In this paper, the development of an ion trap based palm portable mass spectrometer (PPMS) designed for real time CWA field detection and identification is described in detail. A small ion getter pump has been developed for the PPMS. The performance test results demonstrate the promise of the PPMS as a personal mobile chemical analyzer for field verification of chemical warfare agents. Further developments of the PPMS for prospective applications in other areas such as environmental sciences, homeland security, and space explorations are also discussed.

\section{Experimental}

\section{Ion Trap Assembly}

For the PPMS in this experiment, four parallel disks with coaxial holes have been used for the ion trap as shown in Figure 1. This geometry is very simple to manufacture. The optimum dimension of the electrodes can be found through the SIMION simulation.

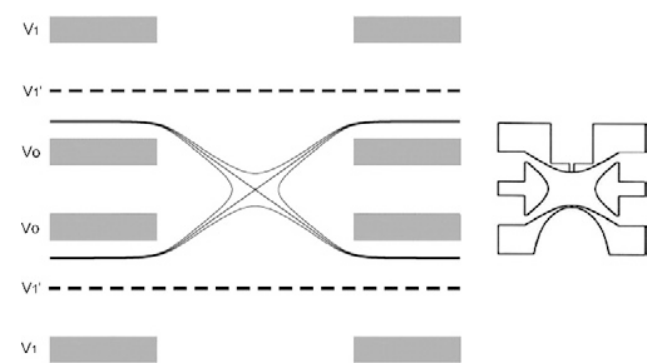

Figure 1. Equipotential surfaces that cross the center of the four-parallel-disk ion trap and adjacent ones simulated by SIMION, alongside a Paul type hyperboloid trap for comparison.

Two ring electrodes with a thickness of $0.3 \mathrm{~mm}$ are separated by $0.5 \mathrm{~mm}$ spacers in this experiment. The distance between ring and end cap electrode is $1 \mathrm{~mm}$. With this separation, the optimum diameter of the coaxial hole is found to be $2.05 \mathrm{~mm}$. If a potential of $\pm 500 \mathrm{~V}$, for example, is applied between ring and end cap electrodes, the equipotential surface that crosses the center point and two adjacent surfaces of $\pm 10 \mathrm{~V}$ are as plotted in Figure 1 . The figure shows that this ion trap is comparable to a Paul hyperbolic trap with a diameter of $0.48 \mathrm{~mm}$ and applied potential of $\pm 10 \mathrm{~V}$. If the end cap electrode is made of mesh and placed at the halfway point represented by the dotted line in Figure 1, then the applied voltage can be reduced to a half $\left(\mathrm{V}_{1}^{\prime}= \pm 250 \mathrm{~V}\right)$ while generating the same potential distribution as before. This reduces the power load required for $\mathrm{rf}$ high voltage generation. In this experiment, the end cap electrode is a flat disk with a hole of $0.5 \mathrm{~mm}$ diameter and is separated by 0.5 $\mathrm{mm}$ from the ring electrode.

The required rf frequency and voltage for ion ejection can be estimated by an instability equation generally used for Paul type ion traps [16].

$$
\mathrm{q}_{\mathrm{z}}=8 \mathrm{eV} / \mathrm{mr}_{\mathrm{o}}^{2} \Omega^{2},\left(\mathrm{q}_{\mathrm{z}}=0.908\right)
$$

where $\mathrm{e} / \mathrm{m}$ is the charge to mass ratio, $\mathrm{r}_{\mathrm{o}}$ is the radius, and $\Omega$ is the frequency of rf voltage $V$. V denotes peak voltage. The mass to charge ratio, $m / z$, at the instability boundary is given by

$$
\mathrm{m} / \mathrm{z}=8 \mathrm{~V} /\left(0.908 \mathrm{r}_{\mathrm{o}}^{2} \Omega^{2}\right) .
$$

For the example described above, the value $\mathrm{V} / \mathrm{r}_{\mathrm{o}}^{2}$ is

$$
\mathrm{V} / \mathrm{r}_{\mathrm{o}}^{2}=10 /(0.24)^{2}=173.6\left(\mathrm{~V} / \mathrm{cm}^{2}\right) .
$$

The required rf frequency $\Omega$ for ejection of singly charged ions of mass $\mathrm{m}$ can be obtained from the equation

$$
\mathrm{m} / \mathrm{z}=1529 / \Omega^{2} \text {. }
$$

If $\Omega=3.9 \mathrm{MHz}$, then

$$
\mathrm{m} / \mathrm{z}=100 .
$$

This means that $500 \mathrm{~V}_{p-p}$ at $3.9 \mathrm{MHz}$ ejects ions of $\mathrm{m} / \mathrm{z}$ 100 from the four-parallel-disk ion trap and rf voltage of $1500 \mathrm{~V}_{p-p}$ is required to scan ions of $m / z 300$. 
All the electrodes are made of stainless steel and the ceramic spacers are manufactured with a tolerance within $0.01 \mathrm{~mm}$. A standard electron gun with a rhenium filament is mounted on top of the ion trap. The electron current is precisely controlled and focused into the ion trap. A micro-channel plate ion detector with a collection diameter of $5.5 \mathrm{~mm}$ has been mounted at the other end of the ion trap. Figure 2 shows a picture of an ion trap assembly with a diameter of $16 \mathrm{~mm}$ and height of $42 \mathrm{~mm}$, mounted on an UHV electrical feedthrough with a 1.33 in. flange.

\section{High Vacuum System}

A home-made ion getter pump has been developed with a diameter of $50 \mathrm{~mm}$ and height of $35 \mathrm{~mm}$. Since it functions only at a gas pressure below $10^{-3}$ torr, the vacuum chamber needs to be pre-evacuated by a roughing pump. Once evacuated, the roughing pump is detached and the ion getter pump alone maintains high vacuum of up to $10^{-7}$ torr. Its pumping speed is $2 \mathrm{~L}$ per $\mathrm{s}$ and it draws a power current of $2 \mathrm{~mA}$ at a gas pressure of $10^{-4}$ torr and only a few tens of micro-amperes at the $10^{-6}$ torr range. Since the discharge current depends on gas pressure, the current reading can be an indication of vacuum pressure. Measurements of the wear rate of titanium cathode in the ion getter pump indicate an estimated life time of $24,000 \mathrm{~h}$ at $10^{-6}$ torr. This means that the ion getter pump is expected to work for $8000 \mathrm{~h}$, sampling at 1 min intervals.

Figure 3 depicts mass spectrometer hardware components including the ion trap chamber, ion-getter pump, power supply, and pulsed sampling valve. This hardware assembly weighs only $520 \mathrm{~g}$. This weight can be reduced further by modifying the heavy vacuum components.

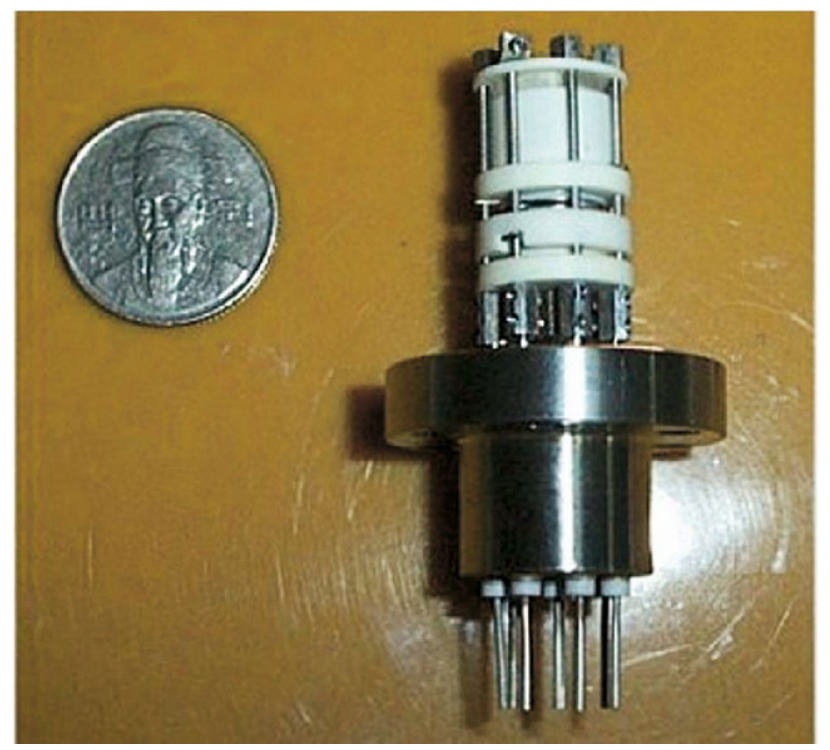

Figure 2. Ion trap assembly with electron gun ionizer and MCP detector, mounted on a UHV electrical feedthrough with 1.33 in. flange.

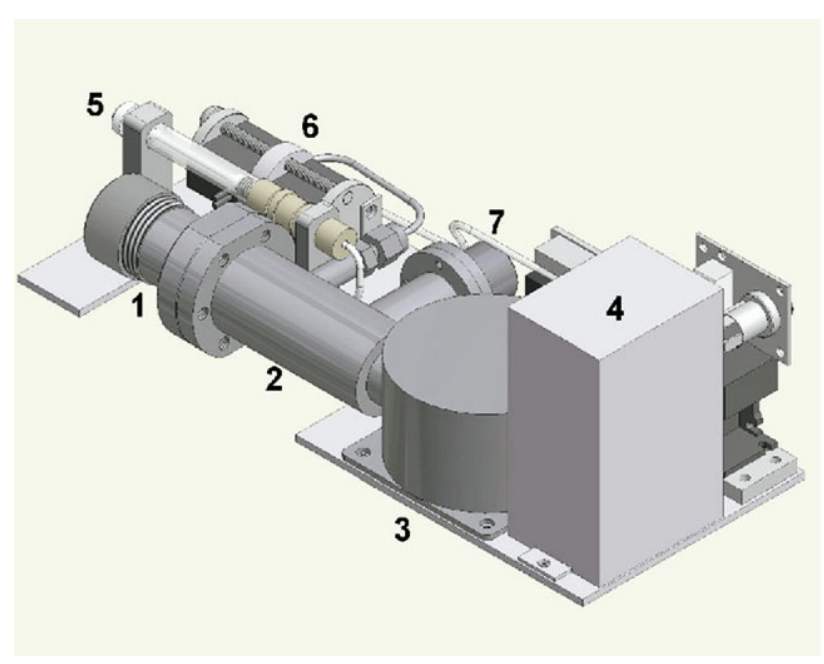

Figure 3. PPMS hardware that weighs 520 g. (1) ion trap assembly, (2) vacuum chamber, (3) ion getter pump, (4) power supply for the ion-getter pump, (5) air inlet, (6) pulsed sampling valve, (7) initiation vacuum valve for pre-evacuation.

\section{Pulsed Gas Sampling}

Air samples were introduced in pulse mode. Pulsed sample gas introduction reduces the pumping load of the ion getter pump and minimizes contamination of the chamber by chemical agents. A pulsed micro valve has been developed that passes exactly the same volume of air in each opening. The volume of air introduced through the valve is $0.034 \mathrm{~mm}^{3}$ and the tolerance is less than $1 \%$, as shown in Figure 4. This volume of air increases the gas pressure up to a peak of $2.5 \times 10^{-4}$ torr. After $1 \mathrm{~s}$, pressure falls to $3 \times 10^{-6}$ torr, and after $5 \mathrm{~s}$, the vacuum reduces to $10^{-6}$ torr, enabling the PPMS to measure a new sample gas every $5 \mathrm{~s}$.

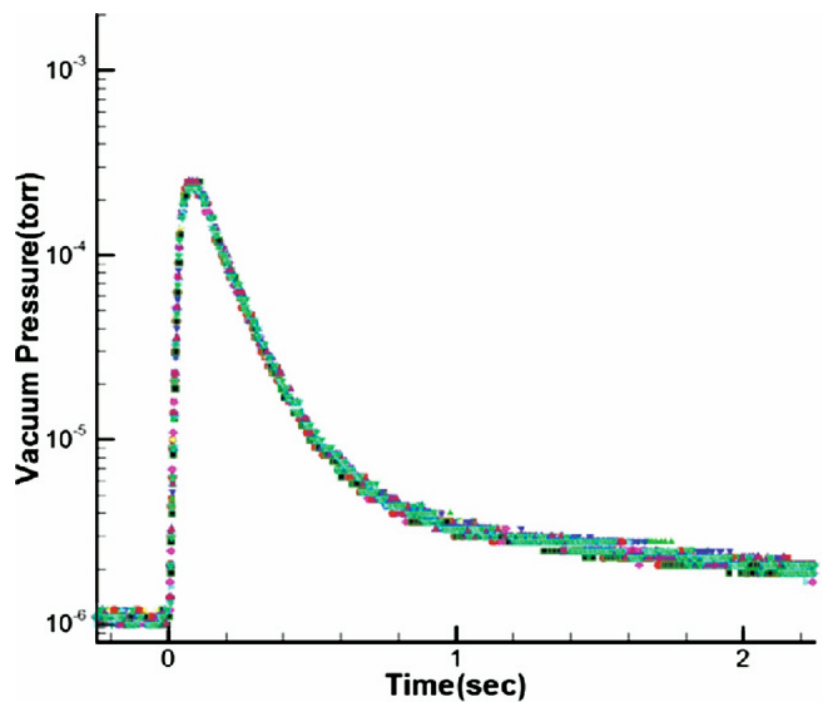

Figure 4. The decrease in vacuum pressure over time in the ion trap chamber from opening the pulsed sampling valve. Over 10 measurements, the tolerance of the introduced air volume was less than $1 \%$. 


\section{Control Electronics}

The control electronics consists of two printed circuit boards. An analog circuit board provides various DC voltages converted from a battery, generates rf high voltage for mass scanning, and manipulates measured ion signals. The digital circuit board is an embedded micro computer with a CPU ARM core that sets up operation parameters, diagnoses the functions, and executes measurement. The electron gun draws a maximum current of $3 \mathrm{~A}$ at $2 \mathrm{~V}$. However, it turns on only during the mass scan to save battery power.

A rf sine wave is generated by a direct digital synthesizer (DDS) and multiplied by a mass scan function generated by the CPU. Then it is amplified and transformed to $1500 \mathrm{~V}_{p-p}$ by an impedance matching transformer. The detected ion signal is amplified, filtered, and digitized by an analog/digital converter with a speed of 450 KPS in 16 bit resolution. A TFT liquid crystal display is mounted for spectrum display and touch screen keyboard input. A lithium polymer battery, SLB603870H, delivers a voltage of $3.7 \mathrm{~V}$ with a power capacity of $1500 \mathrm{mAh}$. Ten of these in two parallel stacks deliver $3000 \mathrm{mAh}$ at $18.4 \mathrm{~V}$. Four DC/DC converters deliver voltages of $\pm 5 \mathrm{~V}, \pm 15 \mathrm{~V},-200 \mathrm{~V}, 100$ $\mathrm{V},-2000 \mathrm{~V}$, and $3800 \mathrm{~V}$. The system power draw peaks at $40 \mathrm{~W}$ during measurement, but drops to $3 \mathrm{~W}$ when idle. The idle state means that the electron gun does not emit electrons and the vacuum is below $10^{-6}$ torr, so that the ion getter pump draws only a few tens of microamperes. Since the electron gun and rf HV generator draw the peak current for only a very short time period, making one measurement per minute draws 5 $\mathrm{W}$ on average. At that rate, the PPMS can operate for $10 \mathrm{~h}$ using a battery of $3000 \mathrm{mAh}$.

\section{Software}

The PPMS unit communicates with a personal computer through a USB cable. File management and scan functions are programmed in the PC and loaded in the PPMS memory. These functions include:

1. Individual hardware control—controls all ports and pins of the hardware parts.

2. Vacuum system control-this allows the system to start only when the vacuum is ready.

3. Self diagnosis-checks all major hardware functions. When all parameters are normal, the system becomes ready and waits for command. Otherwise it diagnoses the malfunction and displays instructions.

4. Mass spectrum measurement-executes a sequence of actions including opening the sampling valve, activating the electron gun and ion detector high voltage, generating rf high voltage, starting the scan function, and recording the mass spectrum.
5. Result display-displays ion signals as raw signals in time scale, analog spectrum in mass scale, or digitized ion peak bars in mass scale.

6. Identification from data library-compares measured ion peak patterns with a data library, identifies the specific chemical substance, and displays the chemical name and concentration level.

7. Alarm - when the detected chemical substance is recognized as a threatening agent, an alarm either flashes or emits a sound.

8. Background noise subtraction-regular background patterns and random noise are registered and subtracted.

9. Spectrum average-repeated mass spectra can be averaged.

10. Parameter setting-measurement parameters can be set up and modified by touch-screen input.

11. Data processing-measured mass spectra are manipulated by the PC for various purposes.

12. Data library register-standard mass spectra of chemical substances are registered in the data library for future pattern recognition.

\section{Mass Spectrum Measurement}

Mass spectrum measurement starts with a keyboard executed command to generate a synchronization pulse. About $100 \mathrm{~ms}$ after sample valve opening, when the pressure of the chamber begins to drop, a sequence of scan functions begins followed by activation of the electron gate, activation of the high voltage ion detector, rf mass scan, and ion signal processing. Ion trapping time is variable from 1 to $100 \mathrm{~ms}$ and mass scan time is variable from 10 to $30 \mathrm{~ms}$. Mass spectrum measurement can be repeated up to eight times in one sampling. Scan mass range is variable up to $\mathrm{m} / \mathrm{z} 300$. Low mass ions (below $\mathrm{m} / \mathrm{z} 45$ ) are excluded to eliminate background air ions. No extra buffer gas is used.

The measured ion signal is amplified, digitized, and saved into the memory. The spectrum can be displayed as either a raw ion signal in time scale or in mass scale, or as a mass peak bar integrated for a mass unit. The peak pattern of measured mass spectrum is compared with the data library and if identified as a threatening agent, an alarm begins either to flash or to issue a sound. The measured data is transferred to a PC through a USB cable for further processing.

\section{Results}

The final shape of the PPMS integrating all the components in a palm portable size is shown in Figure 5. The dimensions are $8.2 \times 7.7 \times 24.5 \mathrm{~cm}^{3}$ $(1.54 \mathrm{~L})$ and the weight is $1.48 \mathrm{~kg}(3 \mathrm{lb})$. The battery weight is excluded since $200 \mathrm{~g}$ of lithium polymer battery delivers $1500 \mathrm{mAh}$, while $400 \mathrm{~g}$ delivers 3000 mAh. A fully charged battery of $3000 \mathrm{mAh}$ can operate the PPMS for $10 \mathrm{~h}$. 


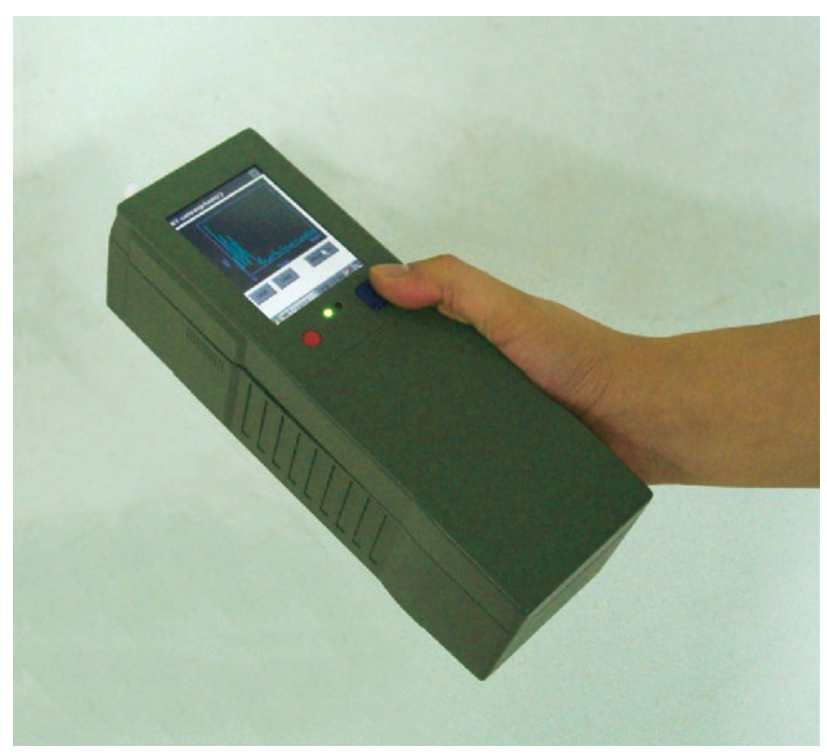

Figure 5. Completed final shape of the PPMS.

Performance testing of the PPMS has been carried out by the Korea Research Institute of Standard and Sciences (KRISS). The standard sample gas was carefully generated by mixing primary reference materials in a standard environment, according to ISO6142. Various concentrations of benzene, toluene, xylene, tetrachloroethylene (TCE), methyl salicylate (MeS), and dimethyl-methylphosphonate (DMMP) have been used for measurement of mass spectra, linearity, detection limit, and reproducibility. Since real chemical warfare agents are not allowed in a civil laboratory, DMMP and $\mathrm{MeS}$ are used for nerve agent simulants and TCE for a choking agent simulant. The detection limit of the PPMS has been measured as $6.4 \mathrm{ppm}$ for toluene and $52.9 \mathrm{ppm}$ for TCE in the air. Figure 6 shows the mass spectra of DMMP diluted at $49.6 \mathrm{ppm}$ in the air.

\section{Discussion}

\section{Four-Parallel-Disk Ion Trap Geometry}

The four-parallel-disk ion trap has proven to be a useful design for miniaturization of a mass spectrometer both in manufacturing and interpretation of the instability diagram. The internal diameter of $2.05 \mathrm{~mm}$ seems to be appropriate for a palm portable mass spectrometer. Since the electric field at the center is virtually linear along the $r-$ and $z$ axes as that of a hyperboloid ion trap, the instability diagram from the solution of the Mathieu equation is appropriate. The relationship of rf voltage, frequency, and ion mass has been experimentally confirmed to correspond very well to that of the instability equation. The electric field in a hyperboloid ion trap is linear on the $\mathrm{z}$ axis. However, the electric field very close to the end cap electrode in this geometry becomes constant. This may result in easier ion cooling without a buffer gas. The effect of nonlinearity at the boundary is to be investigated in more detail. The four-parallel-disk ion trap should be useful for two further experiments. First, mounting the electron gun on one side and two ion detectors on the ends of the ion trap enables one to simultaneously measure both negative and positive ion mass spectra. Second, optical spectra of trapped ions can be measured using resonant laser excitation through the gap between the two ring electrodes. Measurements of both optical spectrum and mass spectrum should certainly enhance the specificity of unknown chemical substances. These are of great interest for future ion trap experiments.

\section{Miniaturized High Vacuum System}

Ion-getter pumps have been found to be well suited for a miniaturized mass spectrometer. There is no internal mechanical motion, enhancing durability, and it draws a low power current, extending battery life. One disadvantage is that it only operates below $10^{-3}$ torr, necessitating a prevacuum pump when the system starts from atmospheric pressure. However, it does not need a prevacuum pump after having been once evacuated. We were concerned that the ion pump may again need an auxiliary pump when restarting after having been shut down for a long time. However, the home-made ion-getter pump restarts alone very well even after a shut down of $3 \mathrm{~d}$ for storage. This means that if the PPMS is turned on and its system is evacuated for 10 min every $3 \mathrm{~d}$, the ion-getter pump alone can keep the system working without an auxiliary vacuum pump.

\section{Pulsed Gas Sampling}

Pulsed sample gas introduction has a number of advantages for portable ion trap mass spectrometry. First, it reduces the load of the high vacuum pump, extending lifetime of the ion-getter pump and saving battery power. Second, it enables one to concentrate target gas ions only in the ion trap. When the sample gases are ionized continuously in the ion trap while air ions are

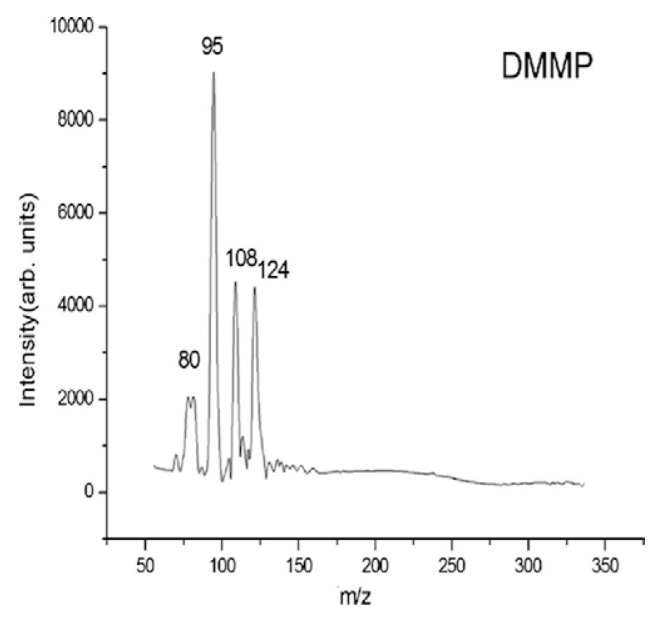

Figure 6. Mass spectra of dimethyl-methylphosphonate diluted in the air at $49.6 \mathrm{ppm}$. 
ejected selectively, there remain only target ions in the ion trap after the pressure pulse.

\section{Mass Spectrometer Functions}

A mass spectrum of mass range up to $m / z 300$ has been demonstrated. The lower ion mass was cut off at $m / z 45$ to eliminate excessive air molecule ions. The mass resolution of three mass units observed would be sufficient for identifying chemical agents as long as the recognition pattern is reproducible. Improvements in mass resolution should be possible as efforts continue to optimize operating parameters.

The demonstrated detection limit was 6.4 ppm for toluene in the air. Since the maximum ion density allowed in an ion trap is about $6 \times 10^{12}$ ions $/ \mathrm{m}^{3}$ [15] because of repulsion forces, the number of ions in the ion trap with $2 \mathrm{~mm}$ i.d. is expected to be about $2 \times 10^{4}$ ions at maximum. If this number of air molecules is ionized and trapped, the probability of finding a target ion in the ion trap would seem to be only 0.1 for the sample concentration of $6 \mathrm{ppm}$. However, detection of target ions at these low concentrations can be achieved by selective ion concentration in the ion trap. Since the low mass cut off is at $m / z 45$ during the ion trapping period, the produced air ions are immediately expelled while the target ions are continuously accumulated. The air sample is introduced in pulse mode and the system is evacuated again in a short time period, so that mainly the target ions remain in the ion trap after the pressure pulse. This selective ion concentration using a pulsed gas sampling is a very useful method for detection of trace gases extremely diluted in the air.

Adding a gas concentrator-like membrane or adsorption/desorption substrate would enhance the detection limit up to a factor of several hundred [17]. However, this addition is not very desirable because those options would extend the detection time to several minutes. The clean-up time of the concentrator would be even longer. Enhancing the efficiency for selective ion accumulation in the ion trap would be a better solution for lower detection limit.

MS/MS would be possible in this ion trap but it has not yet been attempted. Axial modulation in a miniaturized ion trap using a second rf voltage between two end cap electrodes has been experimentally studied [18]. Using a third rf voltage between two ring electrodes is another possibility for axial modulation. Selective ion trapping/ejection for MS/MS using a rectangular wave, instead of a sine wave, has been theoretically studied for this geometry [19]. This report has suggested that selection of ion mass in the ion trap or selective ion ejection would be possible by controlling the duty cycle of the rectangular pulse, instead of additional DC voltage or secondary rf voltage. Since fast switching semiconductors are available nowadays, study of MS/MS with rectangular wave rf scanning is also of future interest.

\section{Usefulness as a Portable Chemical Analyzer}

The PPMS has demonstrated its capability to detect and identify chemical agents in a palm size package, making it the smallest complete mass spectrometer developed so far. Its weight and size make it suitable for use as personal equipment in the battle field. It can be operated for $10 \mathrm{~h}$ continuously with a battery of $400 \mathrm{~g}$. Detection time of less than $1 \mathrm{~s}$ enables one to monitor air contamination from a fast moving vehicle or aircraft. The law enforcement and corrections standards and testing program from the National Institute of Justice has recommended 16 selection factors for evaluation of chemical detection equipment [2]. Table 1 summarizes those factors for the PPMS.

\section{Further Development and Applications}

The size and weight of the PPMS can be reduced by modifying the parts for mass production. Efforts to enhance the detection limit by attaching a gas concentrator at the sampling port or increasing the efficiency

Table 1. Evaluation of the PPMS by 16 selection factors suggested by NIJ [2]

\begin{tabular}{|c|c|}
\hline Evaluation factors & Palm portable mass spectrometer \\
\hline Chemical agent detected-CWA & Detection/identification of DMMP, TCE, MeS demonstrated \\
\hline Chemical agent detected-TIM & Detection/identification of benzene, toluene, xylene demonstrated \\
\hline Detection limit & $6 \mathrm{ppm}$ of toluene and $53 \mathrm{ppm}$ of TCE in the air \\
\hline Resistance to interferants & Specifies chemical substances by ion mass spectrum \\
\hline Response time & $1 \mathrm{~s}$ \\
\hline Start-up time & $10 \mathrm{~min}$. Clean-up time takes $3 \mathrm{~s}$ \\
\hline Detection states & Gas phase only \\
\hline Alarm capability & It takes $1 \mathrm{~s}$ \\
\hline Portability & Size $1.54 \mathrm{~L}$; weight $1.48 \mathrm{~kg}$ (1.88 kg with battery) \\
\hline Power capability & Power consumption $5 \mathrm{~W}$ in average \\
\hline Battery need & $400 \mathrm{~g}$ of battery can operate $10 \mathrm{~h}$ \\
\hline Operational environment & Normal operation confirmed after storage temperature of -10 and $40^{\circ} \mathrm{C}$ \\
\hline Durability & - \\
\hline Procurement cost & - \\
\hline Operator skill level & - \\
\hline Training requirement & - \\
\hline
\end{tabular}


of selective ion concentration should be continued. Detection capability of biological warfare agent using a PPMS represents a further challenge, since this requires pyrolysis of a living organism for fatty acids analysis. Meeting the military standard, MIL-STD 810/461, would be a concern for engineering development.

A small size mass spectrometer has been of much interest in many other areas where mobility of the chemical analyzer is a primary concern for real-time measurement in the field, such as air contamination monitoring and odor investigation in the environmental sciences, the detection of illegal trafficking of drugs and explosives for homeland security, and material analyses for space exploration. However, the PPMS has to be modified in various aspects to meet the operational requirements for those applications, since it has been developed primarily as a CWA detector for military use.

For environmental applications, the target gases would be toxic industrial chemicals. The required detection limit should be of ppb level for foul odor differentiation. Since an ion trap has a limited capacity of trapping ions, an auxiliary device is needed that concentrates the target gases in the air. Thermal adsorption/desorption gas concentrators and gas penetrating membranes have been widely used in the environmental sciences. Attaching a small gas concentrator at the sample inlet port of the PPMS should enhance the detection limit but may also extend the detection time up to several minutes. Further reduction in PPMS size would not be necessary for this application. For homeland security, the operational requirement would not be much different from that for environmental application since the target chemicals are mostly controlled substances such as explosives or drugs.

For space exploration, reduction of size and weight should decrease the expense of a long interplanetary journey, especially when the device has to land on the planet surface with a parachute. The target materials would be atmospheric gases and rock compositions on planet surfaces and frozen material on a comet. A mass resolution high enough to measure isotope ratios of elements would be of primary consideration rather than the detection limit of organic molecules. The PPMS should then shift the mass range and adopt the high resolution techniques demonstrated with a $0.5 \mathrm{~mm}$ ion trap [12]. To analyze solid particles, it needs an evaporation device, a complicated modification. Operational conditions in harsh environments also should be of consideration, such as surrounded gas pressure and extremely low or hot temperatures. An electronic device under gas pressure of glow discharge region as on the Martian surface (about $0.8 \mathrm{kPa}$ ) may not use a potential higher than the discharge breakdown voltage (about $100 \mathrm{~V}$ depending on gas, pressure, and electrodes) because even one shock of discharge breakdown may ruin control electronics. In this case, a small ion trap operated in frequency modulation with a low rf voltage would be one of the solutions for mass spec- trometry. An advanced sampling system is also required where the atmospheric pressure is very high as on the surface of Venus.

For any application where the size of the mass spectrometer is a primary concern, the modification of the PPMS frame would be a good start with the mantra of small, fast, and cheap.

\section{Conclusions}

A palm portable mass spectrometer has been developed with a size of $1.54 \mathrm{~L}$ and a weight of $1.48 \mathrm{~kg}$ with a power consumption of $5 \mathrm{~W}$ in average. Its size and performance results suggest usefulness as a personal mobile chemical analyzer in the field.

\section{Acknowledgments}

The authors acknowledge funding of this project by the Dual Use Technology Center of the Korea Ministry of Defense, contract no. 01-DU-EB-01.

\section{References}

1. Brletich, N. R.; Waters, M. J.; Bowen, Tracy, G. W.; M. F. In Worldwide Chemical Detection Equipment Handbook Chap. 2; Chemical and Biological Defense Information Analysis Center: Gunpowder Branch Aberdeen Proving Ground, MD, 1995.

2. Fatah, A. A.; Barrett, J. A.; Arcilesi, R. D. Jr.; Ewing, K. J.; Lattin, C. H.; Helinski, M. S. In Guide for the Selection of Chemical Agent and Toxic Industrial Material Detection Equipment for Emergency First Responders Chap 3; National Institute of Justice, NIJ Guide 100-00, Law Enforcement and Corrections Standards and Testing Program: Coordinated by Office of Law Enforcement Standards, National Institute of Standards and Technology, Gaithersgburg, MD, June 2000.

3. Hill, H. H. Jr.; Martin, S.J.; Conventional Analytical. Methods for Chemical Warfare Agents. Pure Appl. Chem. 2002, 74, 2281-2291.

4. Eiceman, G.; Karpas, Z. Ion Mobility Spectrometry; CRC Press Inc.: Boca Raton, 1994 , pp. 39-68.

5. Ballantine, D. J. Jr.; Martin, S. J.; Ricco, A. J.; Frye, G. C.; Zellers, E. T.; White, R. M.; Wohltjen, H. Acoustic Wave Sensors: Theory, Design, and Physico-Chemical Applications; Academic Press: San Diego, 1996, 70-98.

6. CBMS BlockII, Oak Ridge National Laboratory, Fact Sheet, July 30, 2002.

7. Gao, L.; Song, Q.; Patterson, G. E.; Cooks, R. G.; Ouyang, Z. Handheld Rectilinear Ion Trap Mass Spectrometer. Anal. Chem. 2006, 78, 5994-6002.

8. PICO, MKS Instruments Inc., http://www.mksinst.com/product/ Product.aspx?ProductID $=286$.

9. An overview of chemicals defined as chemical weapons, http:// www.opcw.org/resp/html/cwagents.html.

10. Henry, C. M. The Incredible Shrinking Mass Spectrometry. Anal. Chem. News Features 1999, 71, (April 1), 265A-268A.

11. Badman, E. R.; Cooks, R. G. Miniature Mass analyzers. J. Mass Spectrom. 2000, 35, 659-671.

12. Kornienko, O.; Reilly, P. T. A.; Whitten, W. B.; Ramsey, J. M. Micro Ion Trap Mass Spectrometry. Rapid Commun. Mass Spectrom. 1999, 13, 50-53.

13. Badman, E. R.; Johnson, R. C.; Plass, W. R.; Cooks, R. G. A Miniature Cylindrical Quadrupole Ion Trap: Simulation and Experiment. Anal. Chem. 1998, 70, 4896-4901.

14. Ouyang, Z.; Badman, E. R.; Cooks, R. G. Characterization of a Serial Array of Miniature Cylindrical Ion Trap Mass Analyzers. Rapid Commun. Mass Spectrom. 1999, 13, 2444-2449.

15. Ouyang, Z.; Gao, L.; Fico, M.; Chappell, W. J.; Noll, R. J.; Cooks, R. G. Quadrupole Ion Traps and Trap Arrays: Geometry Material, Scale, Performance. Eur. J. Mass Spectrom. 2007, 13, 13-18.

16. March, R. E.; Hughes, R. J.; Todd, J. F. J. Quadrupole Storage Mass Spectrometry; John Wiley and Sons: New York, 1989; p. 196.

17. Cotte-Rodríguez, I.; Handberg, E.; Noll, R. J.; Kilgour, D. P. A.; Cooks, R. G. Improved Detection of Low Vapor Pressure Compounds in Air by Serial Combination of Single-Sided Membrane Introduction with Fiber Introduction Mass Spectrometry. Analyst 2005, 130, 679-686.

18. Wells, J. M.; Badman, E. R.; Cooks, R. G. A Quadrupole Ion Trap with Cylindrical Geometry Operated in the Mass-Selective Instability Mode Anal. Chem. 1998, 70, 438-444.

19. Lee, W.-W.; Oh, C. H.; Kim, P.-S.; Yang, M.; Song, K. S. Characteristics of Cylindrical Ion Trap. Int. J. Mass Spectrom. 2003, 230, 25-31. 